\title{
Disparity in Crohn's disease activity between home and clinics is associated with unscheduled hospital visits due to disease flares
}

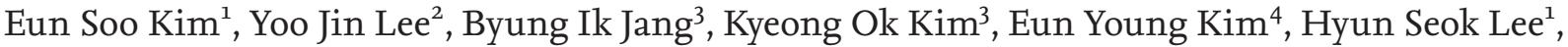 \\ Seong Woo Jeon ${ }^{1}$, Sang Gyu Kwak ${ }^{5}$, and on behalf of Crohn's and Colitis Association in Daegu-Gyeong- \\ buk (CCAiD)
}

\begin{abstract}
${ }^{1}$ Division of Gastroenterology, Department of Internal Medicine, Kyungpook National University School of Medicine, Daegu; ${ }^{2}$ Division of Gastroenterology, Department of Internal Medicine, Keimyung University School of Medicine, Daegu; ${ }^{3}$ Division of Gastroenterology, Department of Internal Medicine, Yeungnam University College of Medicine, Daegu; ${ }^{4}$ Division of Gastroenterology, Department of Internal Medicine, ${ }^{5}$ Department of Medical Statistics, Catholic University of Daegu School of Medicine, Daegu, Korea
\end{abstract}

Received: November 24, 2016 Revised : January 9, 2017

Accepted: April 2, 2017

\section{Correspondence to Byung Ik Jang, M.D.}

Division of Gastroenterology and Hepatology, Department of Internal Medicine, Yeungnam University College of Medicine, 170 Hyeonchung-ro, Nam-gu,

Daegu 42415, Korea

Tel: +82-53-250-8096

Fax: +82-53-250-7088

E-mail:jbi@med.yu.ac.kr

This research was presented in United European Gastroenterology 2015, Barcelona, Spain.
Background/Aims: E-health technologies have been implemented for the management of Crohn's disease (CD). We aimed to identify differences between patient activities at home and at routine clinic visits using a web-based self-reporting $\mathrm{CD}$ symptom diary (CDSD) and to determine the impact of this disparity on clinical outcomes.

Methods: Patients with CD from three tertiary hospitals were invited to assess their symptoms at least once a week using CDSD. We identified patients who showed disparities in disease activity (high activity at home but normal at the next hospital visit) and evaluated clinical outcomes of these patients such as unscheduled visits due to flares using Kaplan-Meier analyses.

Results: One hundred and forty-three patients recorded their symptoms weekly for at least 3 consecutive months and were included. Forty-eight patients (33.6\%) showed disparate disease activities between at home and at the next outpatient clinic visit. The cumulative risk of unscheduled visits was significantly higher in this disparity group than in the concordant group $(p=0.001)$. Disparity in activity $(p=0.003)$, and anti-tumor necrosis factor use $(p=0.002)$ were independent risk factors of unscheduled visits due to disease flares.

Conclusions: Disparity in disease activity is considerable in CD patients and is related to the risk of unscheduled hospital visit.

Keywords: Crohn disease; Disease activity; Mobile technology; Patients reporting outcome

\section{INTRODUCTION}

Crohn's disease (CD) is a chronic inflammatory condition that may affect any part of the gastrointestinal tract [1]. The disease course of CD is extremely unstable and is characterised by its repetitive flares. In fact, more than $90 \%$ of patients with CD experienced relapse within 20 years of their initial diagnosis, indicating the importance of monitoring disease activity [2].

Although the Crohn's Disease Activity Index (CDAI) 
is considered to be the gold standard for evaluation of disease activity, it has been criticised for being complex, impractical, and time-consuming; it is composed of 8 clinical variables requiring laboratory tests for haematocrit and physical examination of variables such as body weight and abdominal mass [3]. As patient symptoms are assessed for the last 7 days, the CDAI may be subject to the risk of recall bias. Furthermore, many key clinical symptoms of disease activity occurring outside of the hospital may not be detected using CDAI during routine clinic visits. Therefore, real-time reporting of disease activity, which may be the ideal method to monitor activity of $\mathrm{CD}$, is needed. In this context, a remote monitoring system using mobile technology could be the perfect solution.

Implementation of self-monitoring with e-health technologies in chronic diseases such as diabetes and congestive heart failure has resulted in improved disease outcomes [4,5]. Several web-based reporting systems have also been developed to manage inflammatory bowel disease (IBD). In a Danish study, an individualised approach to infliximab treatment for $\mathrm{CD}$ using an online reporting system was shown to be practical and feasible [6]. Recently, a novel IBD monitoring index for use with mobile health technologies has been developed and validated [7]. This index was found to identify clinical disease activity with an area under the receiver operating characteristic curve value of 0.9. However, these studies did not determine the significance of the disease activity recorded at home compared with the activity measured in routine outpatient clinic visits. There may be some patients who experienced clinical symptoms such as abdominal pain or diarrhoea at home but did not report them during their routine hospital visit; consequently, their health care providers may not have detected this activity. We hypothesize that this disparity (high activity at home but normal in the next outpatient clinic visit) in reported symptoms may influence a patient's disease course.

We recently developed a web-based self-reporting Crohn's Disease Symptom Diary (CDSD) using the http://www.cdsd.or.kr website and reported that it corresponded well to the CDAI, with a CDSD score of 5 as the cut-off value of clinical remission [8]. Patients can record their symptoms at any moment through a smartphone. The aim of this study was to identify the differenc- es between patients' symptoms recorded at home and during routine hospital visits using the CDSD through mobile technology. We also assessed the impact of this discrepancy on clinical outcomes such as bowel resection surgery, biologics use, and unscheduled visits due to disease flares. Finally, factors associated with a risk of unscheduled hospital visits during the follow-up period were evaluated.

\section{METHODS}

\section{Patients and study design}

This was a prospective multicentre observational ongoing study that evaluated the feasibility and efficacy of the CDSD. Patients from three tertiary referral hospitals in the Daegu-Gyeongbuk area in southeastern Korea who had been followed up for at least 6 months for CD were eligible for inclusion. A diagnosis of CD was made based on a detailed history, physical examination, and a combination of histology, radiographic findings, laboratory tests, and endoscopic figures. Between August 2012 and October 2014, patients with CD were prospectively enrolled and invited to use the CDSD through a mobile phone at http://www.cdsd.or.kr. They were asked to visit the CDSD website and assess their symptoms at home at least once a week. They were also asked to complete a diary entry on the CDSD at their routine outpatient clinic visit. Patients were advised to visit the CDSD on a weekly basis even if they experienced no symptoms (in remission). Demographic characteristics such as age at diagnosis, sex, education level, and marital status and clinical features of CD such as disease location and behaviour were recorded. Data regarding clinical outcomes such as biologics use, bowel resection surgery, and unscheduled visits for disease flares were also obtained during the follow-up period. All patients provided an informed consent. Patients who could not use a smartphone were excluded from the study. If patients did not record their symptoms on a weekly basis for at least 3 consecutive months, they were also excluded. The protocol was approved by the Institutional Review Boards (IRB No. DSMC12-180) of all participating hospitals and was registered in the WHO International Clinical Trials Registry Platform (No. KCTooo0759). 


\section{CDSD website}

The features of the CDSD have been described in detail in a previous study [8]. Briefly, it was composed of five clinical parameters based on the Harvey-Bradshaw index: general well-being, abdominal pain, number of liquid stools per day, abdominal mass, and presence of CD-associated complications. The total score was calculated automatically, with a score of 5 indicating the cut-off value for clinical remission. As the CDSD was designed to be completed online by patients themselves, clinical images of uveitis, erythema nodosum, aphthous ulcer, pyoderma gangrenosum, anal fistula, and abscess were included to help patients better understand the parameters. Symptoms could be recorded by patients anytime and anywhere using a smartphone.

\section{Definitions}

Disparity in activity was defined when the CDSD score at home was higher than the score recorded at the next routine outpatient clinic according to a cut-off value of 5 ( $5 \geq$ at home and $5<$ at outpatient clinic) (Fig. 1A). When patients showed disparity more than $30 \%$ of whole activity record, they were assigned as a disparity group. Con- cordance in activity was defined when the CDSD score at home was in the same category as that recorded at the next routine outpatient clinic, again with a cut-off value of 5. Based on this definition, concordance group was categorised into following three groups: (1) concordance high $(\mathrm{CH})$ : both activities at home and the next outpatient clinic were greater than a CDSD score of 5 (Fig. 1B); (2) concordance low (CL): both activities at home and the next outpatient clinic were equal to or less than a CDSD score of 5 (Fig. 1C); and (3) concordance both (CB): both cycles ( 1 and 2 ) appeared during the entire observation (Fig. 1D). Three authors (E.S.K., B.I.J., and S.W.J.) from each hospital discussed and decided the main pattern of graph for each patient.

An unscheduled visit was indicated when patients unexpectedly had the emergency department (ED) visits, outpatient clinic visits, or unscheduled hospitalization during the follow-up period because of disease flares such as severe abdominal pain, intestinal bleeding, fever, and perianal abscess. When clinical outcomes occurred before patient's main graph pattern was made, they were excluded. Therefore, clinical outcomes were considered only after the observational time frame of
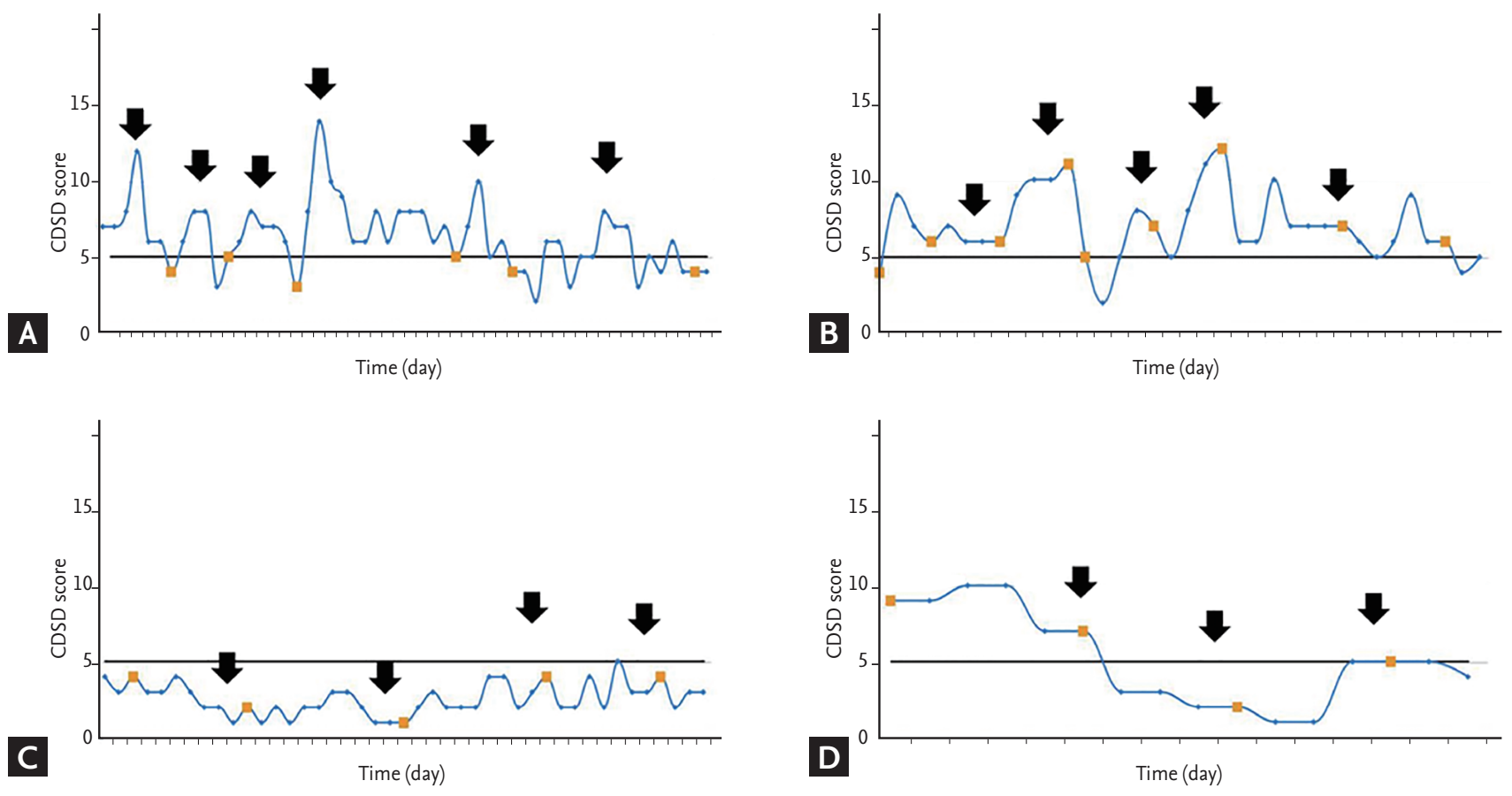

Figure 1. Real patient's graph patterns of different disease activity. (A) Disparity. (B) Concordance high. (C) Concordance low. (D) Concordance both. A black bold line indicates CDSD score 5 as a cut-off value of clinical remission. A square indicates an outpatient clinic visit. Black arrows point the key pattern of the activity graph. CDSD, Crohn's disease symptom diary. 
the graph pattern in each patient.

\section{Statistical analysis}

Summary for characteristics variables were performed using descriptive analysis, the values of mean \pm standard deviation (SD) presented for quantitative variables and the values of frequency (\%) for qualitative variables.

For the univariate analysis of continuous variables, either two sample $t$ test or the Mann-Whitney $U$ test was used as appropriate. For the univariate analysis of categorical variables, chi-square test was used. Kaplan-Meier survival analysis and log-rank or Breslow test were used as survival tools to evaluate clinical outcomes. For the multivariate analysis of factors associated with unscheduled visits due to disease flares, a Cox-proportional hazard ratio was used. Variables that showed a $p$ value $<0.05$ in log-rank or Breslow test were included for multivariate analysis. All tests were two-sided and a $p$ value of $<0.05$ indicated statistical significance. All statistical analyses were performed using SPSS version 18.0 (SPSS Inc., Chicago, IL, USA).

\section{RESULTS}

Of the 280 patients with CD who were invited, 155 (109) men; mean age $26.9 \pm 9.1$ years; median follow-up, 24 months) were included in the study (Fig. 2). Of the 125 patients who were excluded, 106 did not record their activity for at least 3 consecutive months, 15 did not record data, and four refused to participate. Additional 12 patients were excluded because their clinical outcomes occurred before their main activity pattern (disparity or concordance) was determined. Therefore, data of 143 (102 men; mean age, $27.2 \pm 9.3$ years; median follow-up, 20 months) was finally evaluated. Overall, 48 patients (48/143, $33.6 \%$ ) showed discrepant activity (high at home but normal at the next routine outpatient clinic visit), whereas 95 patients $(95 / 143,66.4 \%)$ reported concordant activity. For the concordant group, a large proportion (68/95, 71.6\%) showed low activity (CDSD score $\leq 5)$ both at home and at the hospital (CL), and two patients $(2 / 95,2.1 \%)$ had cycles of high activity (CDSD score $>5$ ) both at home and at the hospital (CH). Twenty-five patients (25/95, 26.3\%) had both concordant cycles of high and low activity at the same time during the observation period (CB).

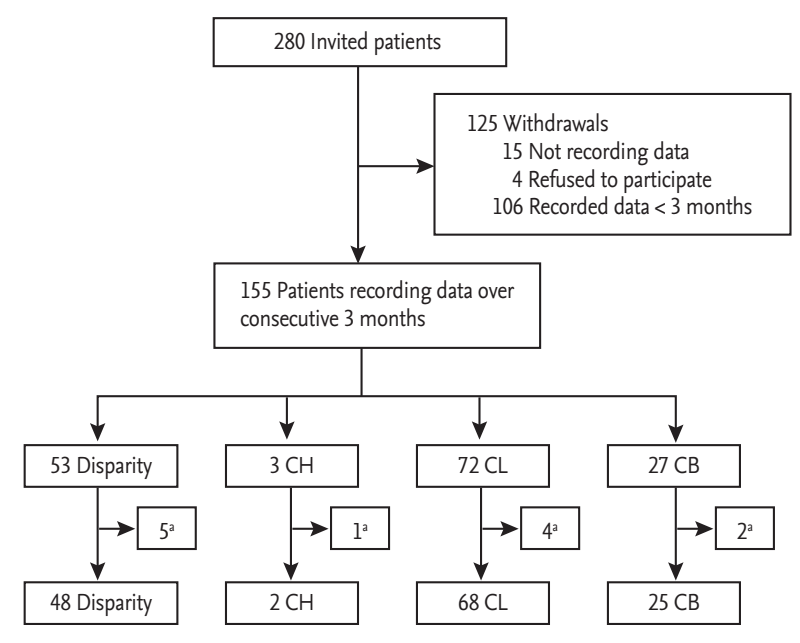

Figure 2. The flow chart of patients participating in the study. CH, concordance high; CL, concordance low; CB, concordance both. ${ }^{a}$ Number of patients whose clinical outcomes occurred before observation period of main graph pattern.

There were no significant differences between disparity and concordance groups regarding baseline characteristics including sex, age, disease duration, education, marital status, disease location, disease behaviour, exposure to steroids, thiopurine use, bowel resection surgery, and biologics use before enrolment (Table 1).

\section{Impact of disparity in activity on clinical outcomes}

The disparity group appeared to have a higher cumulative risk of anti-tumor necrosis factor (TNF) agent use than concordance group although it failed to achieve statistical significance ( $\log$ rank $p=0.114$, Breslow $p=$ o.144) (Fig. 3A). Regarding bowel resection surgery, no significant difference was observed between groups (log rank $p=0.696$, Breslow $p=0.592$ ) and Kaplan-Meier survival graph could not be depicted because of small number of surgery cases during follow-up period. The time from inclusion in the study to an unscheduled visit due to flares was significantly shorter in the disparity group compared with the concordant group $(\log \operatorname{rank} p=0.001$, Breslow $p<0.001$ ) (Fig. 3B).

\section{Risk factors of unscheduled hospital visits}

Next, we estimated the risk factors of unscheduled hospital visits due to disease flares using Kaplan-Meier analysis. In addition to disparity in activity between home and hospital visits, exposure to steroids (log rank 
Table 1. Baseline demographics and characteristics of patients

\begin{tabular}{|c|c|c|c|}
\hline Variable & Disparity $(n=48)$ & Concordance $(\mathrm{n}=95)$ & $p$ value \\
\hline Male sex & $31(64.6)$ & $71(74.7)$ & 0.250 \\
\hline Age at diagnosis, yr & $22.9 \pm 8.3$ & $25.0 \pm 8.7$ & 0.161 \\
\hline Age at inclusion, yr & $25.7 \pm 8.9$ & $27.9 \pm 9.5$ & 0.147 \\
\hline Follow-up duration, mon & $21.9 \pm 10$ & $20.2 \pm 10.2$ & 0.332 \\
\hline Education $\geq$ university & $28(58.3)$ & $69(72.6)$ & 0.110 \\
\hline Marriage & $10(20.8)$ & $29(30.5)$ & 0.183 \\
\hline Disease location & & & 0.410 \\
\hline L1 (ileal) & $17(35 \cdot 4)$ & $26(27 \cdot 4)$ & \\
\hline L2 (colonic) & $8(16.7)$ & $24(25 \cdot 3)$ & \\
\hline L3 (ileocolonic) & $23(47 \cdot 9)$ & $45(47 \cdot 4)$ & \\
\hline Upper gastrointestinal involvement & $2(4.2)$ & $2(2.1)$ & 0.607 \\
\hline Disease behavior at inclusion & & & 0.282 \\
\hline B1 (inflammatory) & $21(43.8)$ & $52(54 \cdot 7)$ & \\
\hline B2 (stricturing) & $9(18.8)$ & $9(9 \cdot 5)$ & \\
\hline B3 (penetrating) & $18(37 \cdot 5)$ & $34(35.8)$ & \\
\hline Perianal disease & $25(52.1)$ & $56(58.9)$ & 0.533 \\
\hline Exposure to steroid & $31(64.6)$ & $52(54 \cdot 7)$ & 0.204 \\
\hline Thiopurine & $45(93.8)$ & $81(85 \cdot 3)$ & 0.124 \\
\hline Previous bowel resection surgery & $10(20.8)$ & $15(15.8)$ & 0.497 \\
\hline Previous biologic use & $8(16.7)$ & $13(13.7)$ & 0.804 \\
\hline
\end{tabular}

Values are presented as number (\%) or mean \pm SD.
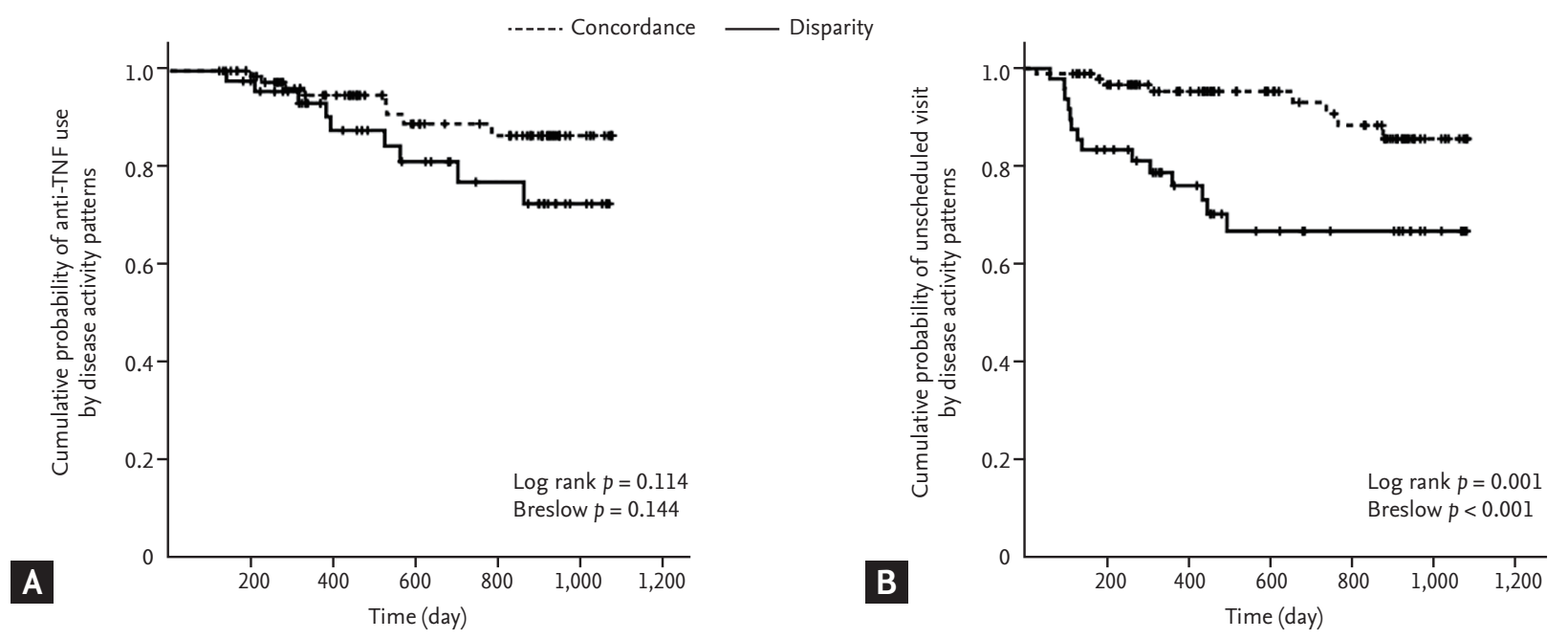

Figure 3. Kaplan-Meier analysis of (A) anti-TNF use, and (B) unscheduled hospital visits according to disparity and concordance pattern of disease activity. TNF, tumor necrosis factor.

$p=0.021$, Breslow $p=0.045)$ (Fig. 4A), anti-TNF use (log rank $p<0.001$, Breslow $p<0.001$ ) (Fig. 4B) and bowel resection surgery after enrolment $(\log$ rank $p=0.001$, Breslow $p=0.011)$ (Fig. 4 C) were significantly related with 

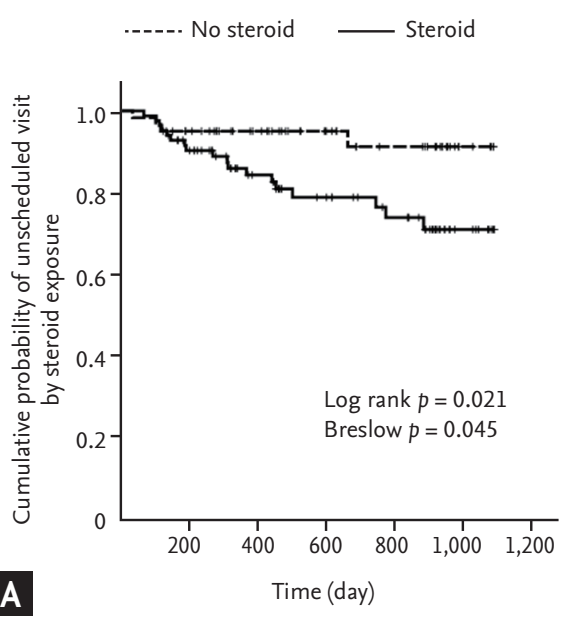

-.-.-. No anti-TNF _ Anti-TNF

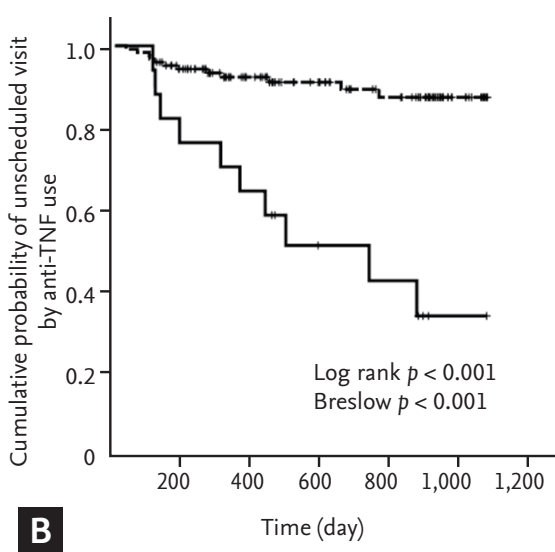

-.-... No surgery Surgery

Figure 4. Kaplan-Meier analysis of unscheduled hospital visits according to (A) steroid exposure, (B) anti-TNF use, and (C) bowel resection surgery. TNF, tumor necrosis factor.

Table 2. Multivariate analysis of the risk factors of the unscheduled hospital visits due to disease flares

\begin{tabular}{lcc}
\hline Risk factor & Hazard ratio (95\% confidence interval) & $p$ value \\
\hline Disparity of activity & $3.82(1.56-9.38)$ & 0.003 \\
Exposure to steroid & $2.62(0.87-7.90)$ & 0.087 \\
Anti-tumor necrosis factor use & $4.34(1.75-10.75)$ & 0.002 \\
Bowel resection surgery & $2.28(0.46-11.24)$ & 0.311 \\
\hline
\end{tabular}

cumulative risk of unscheduled hospital visits. However, there were no significant differences in this risk in terms of sex, education level, thiopurine use, disease behaviour, or disease location. Multivariate Cox proportional hazard analysis revealed that disparity in activity (hazard ratio [HR], 3.82; 95\% confidence interval [CI], 1.56 to $9.38 ; p=0.003$ ), and anti-TNF use after enrolment (HR, 4.34; $95 \%$ CI, 1.75 to $10.75 ; p=0.002$ ) were independent risk factors of unexpected hospital visits due to disease flares (Table 2).

We attempted to evaluate cumulative risk factors of bowel surgery, but failed due to small number of surgery case during follow-up. We found that steroid exposure (log rank $p=0.036)$ and stricturing behaviour (log rank $p=0.010$ ) were cumulative risk factors of anti-TNF use during follow-up period. Among them, the latter remained as an independent risk factor of anti-TNF use in multivariate Cox proportional hazard analysis (HR, 3.98; $95 \% \mathrm{CI}, 1.20$ to $13.27 ; p=0.024$ ) (data not shown).

\section{DISCUSSION}

This prospective observational study with $143 \mathrm{CD}$ patients with a median 20-month follow-up period demonstrated that activity that differed between the home and routine outpatient clinic visits negatively affected the patients' disease outcomes. These findings indicate the importance of robust monitoring of activity when patients with $\mathrm{CD}$ are at home by using mobile technology. To the best of our knowledge, this is the first study to determine the implications of remote monitoring of $\mathrm{CD}$ activity using a smartphone on the prediction of disease course.

Patient-reported outcomes (PRO) have been recognised as a promising measure for evaluating health status in IBD interventions [9,10]. Patient-reported disease activity was shown to be an independent predictor of clinical disease activity in patients with $\mathrm{CD}$, even after including common IBD symptoms such as abdominal pain and stool frequency, similar to the parameters of 
CDSD in the present study $[7,8]$. We confirmed the significance of continuous self-monitoring of disease activity by patients outside of the hospital, which may not be achieved using the traditional CDAI.

Our findings showed that a third of the patients with CD had more than one cycle of high activity while at home that was not detected at their next outpatient clinic visit because they were stable at the hospital. More importantly, these patients had a higher risk of negative outcomes such as unscheduled outpatient clinic visits, ED visits, or unexpected hospitalization due to flares during follow-up. It is evident that when patients complain of symptoms during outpatient clinic visits, we can arrange for the appropriate therapeutic strategy by stepping up medication or shortening the follow-up period for close observation. However, when patients do not report symptoms to their health care providers but still experience activity at home, the delivery of an optimal management plan may occur too late, most likely contributing to unexpected use of health care resources due to disease flares. Similarly, a prospective study evaluating telephone activity at an IBD care centre showed that patients with IBD who made a high volume of telephone calls while staying at home were more likely to have poor clinical outcomes such as more clinic visits, emergency room visits and hospitalisations compared with low telephone users; this suggests that clusters of telephone calls are a red flag that can be used to identify IBD patients at risk for increased health care use and negative clinical outcomes [11]. Similarly, the disparity group in the present study may have represented patients who would need to make frequent phone calls while staying at home.

Non-routine health care visits including ED visits are recognised as a parameter of poor clinical outcomes and health care utilisation in IBD research [11-14]. One of the benefits of telemedicine would be to provide continued care based on sustained monitoring, which may lead to a reduction in unscheduled hospital visits [15]. Hence, in the present study, we sought to identify risk factors of unscheduled visits for disease flares. We found that disparity in activity (HR, 3.82; 95\% CI, 1.56 to 9.38; $p=0.003$ ) was an independent risk factor of increased health care resource use (Table 2). Therefore, we suggest that disparity in activity observed by remote monitoring can play a role as a surrogate marker to detect patients with $\mathrm{CD}$ who are at a high risk of clinical deterioration and increased use of health care resources. Identifying high-risk patients with $\mathrm{CD}$ early by using real-time monitoring and subsequently providing appropriate management may reduce unscheduled hospital visits. The other risk factor found in this study was anti-TNF use (HR, 4.34; 95\% CI, 1.75 to $10.75 ; p=0.002$ ). This result is consistent with a prospective cohort study identifying predictors of hospitalisation and outpatient visits in patients with IBD, supporting the idea that biologics use is a significant predictor of these poor clinical outcomes in CD patients [12].

Apart from disparity group, one might think that patients who showed high activity at home or hospital (concordance high, $\mathrm{CH}$ or concordance both, $\mathrm{CB}$ ) might have a potential risk of poor clinical outcomes like unscheduled hospital visits. Subgroup analysis among concordance groups showed no difference in the cumulative risk of unscheduled hospital visit (log rank $p$ $=0.487$ ) during follow-up. No difference in this risk in subgroup analysis can be explained by following several factors. Too small cases of subgroups might be the probable reason; $\mathrm{CH}$ group had only two cases (Fig. 2). Although concordance group had a high activity, they could get an attention of physicians because this activity was detected at the hospital. Hence, they were probably able to be followed more closely by short-term period interval and be provided with appropriate treatments like step-up therapy, leading to less risk of unscheduled hospital visits.

Several limitations of the study should be noted. First, a large number of patients (37.8\%) were excluded after enrolment in the study due to a lack of sustained participation for at least 3 months. The high attrition rate (up to $60 \%$ ), or low compliance is one of the obstacles faced by studies that use telemedicine in IBD $[16,17]$. We believe that this low compliance might be partly attributed to some difficulty to use telemonitoring system. Therefore, it is needed to simplify and make the system user-friendly. However, when we compared the baseline characteristics of the included patients who recorded more than consecutive 3 months with the patients who were excluded, there were no significant differences except for disease location. There were more L1-type patients (ileal location) among those who recorded their activity for more than 3 months than in those who did 
Table 3. Comparison of baseline characteristics of patients who recorded more than consecutive 3 months with patients excluded from the study

\begin{tabular}{|c|c|c|c|}
\hline Variable & Included patients $(\mathrm{n}=155)$ & Excluded patients $(n=125)$ & $p$ value \\
\hline Age at diagnosis $<18$ years & $41(26.5)$ & $36(28.8)$ & 0.688 \\
\hline Male sex & $109(70.3)$ & $94(75 \cdot 2)$ & 0.420 \\
\hline Education $\geq$ university & $105(67.7)$ & $80(64.0)$ & 0.528 \\
\hline Marriage & $42(27.1)$ & $31(24.8)$ & 0.684 \\
\hline Disease location & & & 0.037 \\
\hline L1 (ileal) & $45(29.0)$ & $21(16.8)$ & \\
\hline L2 (colonic) & $34(21.9)$ & $38(30.4)$ & \\
\hline L3 (ileocolonic) & $76(49.0)$ & $66(52.8)$ & \\
\hline Upper gastrointestinal involvement & $4(2.6)$ & $5(4.0)$ & 0.736 \\
\hline Disease behavior & & & 0.064 \\
\hline Bı (inflammatory) & $76(49.0)$ & $55(44.0)$ & \\
\hline B2 (stricturing) & $20(12.9)$ & $29(23.2)$ & \\
\hline B3 (penetrating) & $59(38.1)$ & $41(32.8)$ & \\
\hline Perianal disease & $88(56.8)$ & $67(53.6)$ & 0.630 \\
\hline Exposure to steroid & $92(59 \cdot 4)$ & $76(60.8)$ & 0.902 \\
\hline Thiopurine & $136(87.7)$ & $105(84.0)$ & 0.390 \\
\hline Previous bowel resection surgery & $27(19.2)$ & $24(19.2)$ & 0.756 \\
\hline Previous biologics use & $21(13.5)$ & $18(14.4)$ & 0.864 \\
\hline
\end{tabular}

Values are presented as number (\%).

not (29\% vs. $16.8 \%, p=0.037$ ) (Table 3). Although the exact cause for this difference is unclear, patients with $\mathrm{L} 1$ may be more likely to pay attention to their symptoms because the ileal location is known to be associated with poor disease course $[18,19]$. This aspect should be taken into account when conducting studies on PRO using telemedicine in patients with CD. Second, patients in remission may be less likely to complete the home diary entries; thereby, might have been excluded from the study. Third, patients in this study may have had a more serious disease course because this study was conducted in tertiary referral hospitals. Thus, care should be taken in the generalisation of these findings. Finally, we could not find the relationship of disease activity patterns with the risk of bowel resection surgery, one of the wellknown disabled outcomes of CD. This might be attributed to the small sample size and relatively short follow-up period to have enough cases of surgery in this study.

In summary, there were considerable differences between the activity reported at home and during hospital visits in patients with $\mathrm{CD}$. This disparity had a negative impact on the disease outcome such as unscheduled hospital visits due to disease flares. These findings suggest that disparity in activity, observed using real-time monitoring through mobile technology, could play a role as an indicator to identify patients at risk of unscheduled hospital visits. Further studies are needed to determine if early detection of unstable activity through continuous monitoring can improve patient disease outcomes.

\section{KEYMESSAGE}

1. A self-reporting symptom monitoring using a smart phone can find that a third of patients with Crohn's disease have a different activity between the home and routine outpatient clinic visits, which may not have been detected using the traditional Crohn's Disease Activity Index.

2. This disparity of disease activity is associated with the poor clinical outcomes such as bowel resection surgery and unscheduled hospital visits due to disease flares. 


\section{Conflict of interest}

No potential conflict of interest relevant to this article was reported.

\section{Acknowledgments}

This work was supported by Basic Science Research Program through the National Research Foundation of Korea (NRF) funded by the Ministry of Education (2015R1D1A1A02062168).

\section{REFERENCES}

1. Vermeire S, van Assche G, Rutgeerts P. Review article: altering the natural history of Crohn's disease: evidence for and against current therapies. Aliment Pharmacol Ther 2007;25:3-12.

2. Veloso FT, Ferreira JT, Barros L, Almeida S. Clinical outcome of Crohn's disease: analysis according to the Vienna classification and clinical activity. Inflamm Bowel Dis 2001;7:306-313.

3. Sands BE, Ooi CJ. A survey of methodological variation in the Crohn's disease activity index. Inflamm Bowel Dis 2005;11:133-138.

4. Haas L, Maryniuk M, Beck J, et al. National standards for diabetes self-management education and support. Diabetes Care 2013;36 Suppl 1:S100-S108.

5. Heneghan C, Ward A, Perera R, et al. Self-monitoring of oral anticoagulation: systematic review and meta-analysis of individual patient data. Lancet 2012;379:322-334.

6. Elkjaer M, Shuhaibar M, Burisch J, et al. E-health empowers patients with ulcerative colitis: a randomised controlled trial of the web-guided 'Constant-care' approach. Gut 2010;59:1652-1661.

7. Van Deen WK, van der Meulen-de Jong AE, Parekh NK, et al. Development and validation of an inflammatory bowel diseases monitoring index for use with mobile health technologies. Clin Gastroenterol Hepatol 2016;14:17421750.

8. Kim ES, Park KS, Cho KB, et al. Development of a webbased, self-reporting symptom diary for Crohn's disease, and its correlation with the Crohn's Disease Activity Index: web-based, self-reporting symptom diary for Crohn's Disease. J Crohns Colitis 2017;11:1449-1455.
9. Jensen RE, Rothrock NE, DeWitt EM, et al. The role of technical advances in the adoption and integration of patient-reported outcomes in clinical care. Med Care 2015;53:153-159.

10. Williet N, Sandborn WJ, Peyrin-Biroulet L. Patient-reported outcomes as primary end points in clinical trials of inflammatory bowel disease. Clin Gastroenterol Hepatol 2014;12:1246-1256.

11. Ramos-Rivers C, Regueiro M, Vargas EJ, et al. Association between telephone activity and features of patients with inflammatory bowel disease. Clin Gastroenterol Hepatol 2014;12:986-994.

12. Sulz MC, Siebert U, Arvandi M, et al. Predictors for hospitalization and outpatient visits in patients with inflammatory bowel disease: results from the Swiss Inflammatory Bowel Disease Cohort Study. Eur J Gastroenterol Hepatol 2013;25:790-797.

13. Nguyen GC, Sheng L, Benchimol EI. Health care utilization in elderly onset inflammatory bowel disease: a population-based study. Inflamm Bowel Dis 2015;21:777-782.

14. Gajendran M, Umapathy C, Loganathan P, Hashash JG, Koutroubakis IE, Binion DG. Analysis of hospital-based emergency department visits for inflammatory bowel disease in the USA. Dig Dis Sci 2016;61:389-399.

15. Aguas Peris M, Del Hoyo J, Bebia P, et al. Telemedicine in inflammatory bowel disease: opportunities and approaches. Inflamm Bowel Dis 2015;21:392-399.

16. Cross RK, Cheevers N, Rustgi A, Langenberg P, Finkelstein J. Randomized, controlled trial of home telemanagement in patients with ulcerative colitis (UC HAT). Inflamm Bowel Dis 2012;18:1018-1025.

17. Cross RK, Jambaulikar G, Langenberg P, et al. Telemedicine for patients with inflammatory bowel disease (TELEIBD): design and implementation of randomized clinical trial. Contemp Clin Trials 2015;42:132-144.

18. Lazarev M, Huang C, Bitton A, et al. Relationship between proximal Crohn's disease location and disease behavior and surgery: a cross-sectional study of the IBD Genetics Consortium. Am J Gastroenterol 2013;108:106-112.

19. Gathungu G, Kim MO, Ferguson JP, et al. Granulocytemacrophage colony-stimulating factor autoantibodies: a marker of aggressive Crohn's disease. Inflamm Bowel Dis 2013;19:1671-1680. 